\section{Derivatization of Glass and Polypropylene Surfaces}

This unit describes the derivatization of solid support media with a versatile linker system. The procedure permits the production of various linker types whose characteristics can be tailored to the requirements of the eventual application. The protocols described here have been used extensively on both nonporous (glass, polypropylene foil) and porous (nylon or polypropylene membrane) substrates. If required, a dendrimeric structure can be formed, thereby increasing the loading capacity of the surface in a stepwise fashion, which is especially useful for glass surfaces.

A prerequisite for derivatization is the presence of anchoring groups (hydroxyl or amino groups) on the support surface. These anchoring groups may be intrinsic to the medium (e.g., amino functions on nylon) or may be introduced by chemical modification. Standard modifications include silanization reactions on glass (see Support Protocol 1) or plasma-amination on polypropylene. The latter process is a standard surface modification technique in the plastics industry. The authors obtained plasma-aminated material from AIMS Scientific Products, which processed polypropylene foils provided by the authors.

Generally, the linker synthesis described below (see Basic Protocol) consists of a two-step process (Fig. 12.4.1) that may be repeated in an iterative way. In a first step, the anchoring groups on the surface are activated by an acylation reagent (e.g., acryloyl chloride, 4nitrophenyl chloroformate). Subsequently, in a second step, these activated intermediates are reacted with an amine component. Preferably, a polyamine is employed, since this increases the surface loading. By careful selection of the polyamine component, the surface properties (e.g., linker length, hydrophobicity, charge) may be customized and adapted to specific requirements. The performance of the derivatization process can be

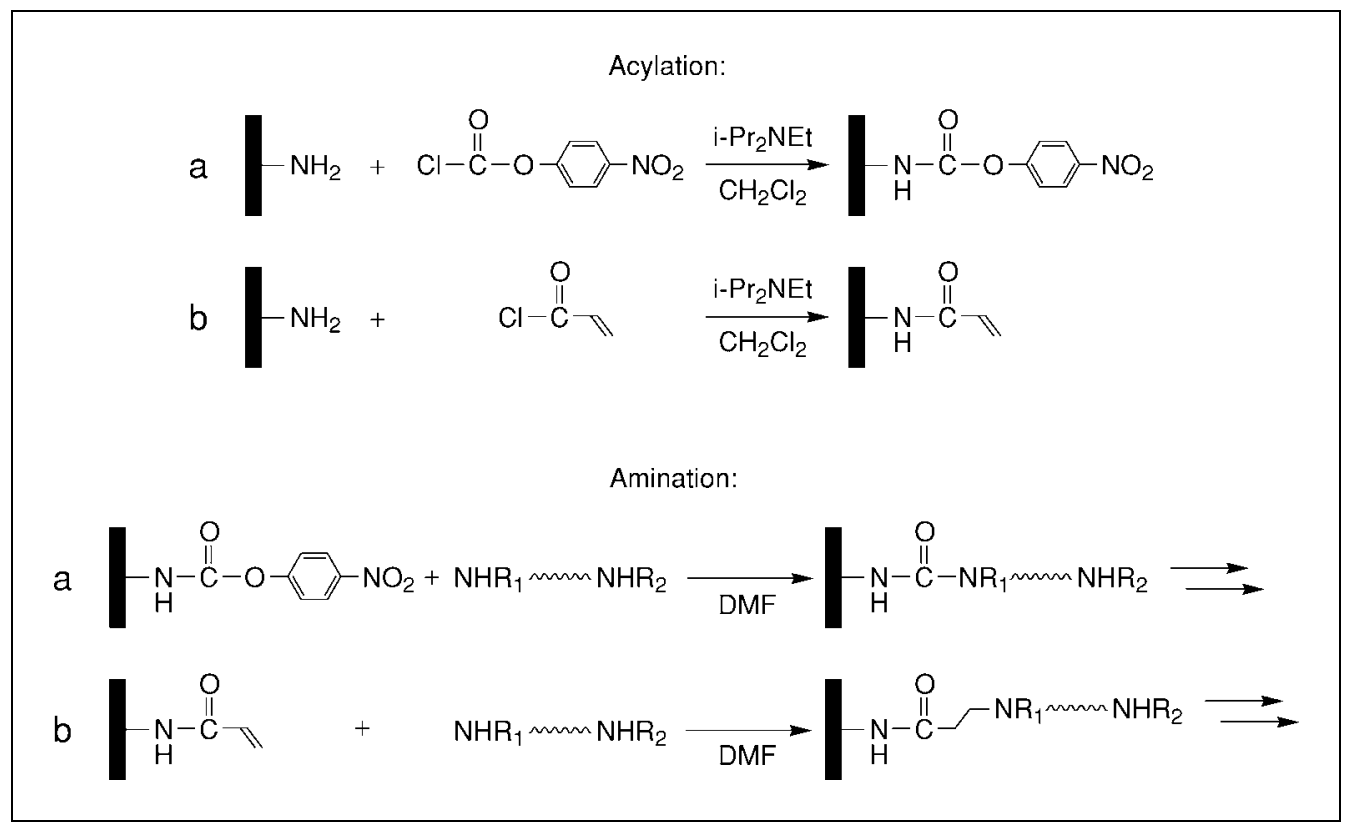

Figure 12.4.1 Linker synthesis on an aminated support. A two-step process of acylation (activation; top) and reaction with various amines (bottom) is used to derivatize the solid support with an appropriate linker. The process is depicted using activation with (a) 4-nitrophenyl chloroformate and (b) acryloyl chloride.

Contributed by Markus Beier and Jörg D. Hoheisel

Current Protocols in Nucleic Acid Chemistry (2004) 12.4.1-12.4.8

Copyright $(C) 2004$ by John Wiley \& Sons, Inc.
UNIT 12.4
Nucleic

Acid-Based

Microarrays and Nanostructures

\subsection{1}

Supplement 18 
monitored by analyzing the density of amino functions on the surface via bromphenol blue staining (see Support Protocol 2).

For the immobilization of existing nucleic acids, an additional activation step is required to generate a covalent linkage between biomolecule and linker. For that purpose, bifunctional cross-linking reagents are used (see Support Protocol 3). In situ synthesis of DNA microarrays does not require such activation but can proceed directly, since the terminal groups of the linker serve as starting points for oligonucleotide synthesis.

NOTE: Use deionized, distilled water in all recipes and protocol steps. Use dry reagentgrade solvents for all reactions.

BASIC PROTOCOL

Derivatization of Glass and Polypropylene Surfaces

\subsection{2}

\section{DERIVATIZATION OF SOLID SUPPORT}

The process described below permits the modification of glass and polypropylene surfaces forming a linker of dendrimeric structure. The derivatization is used either for attaching prefabricated DNA-oligonucleotides, PCR products, and peptide nucleic acid (PNA) oligomers, or for the in situ synthesis of DNA microarrays (UNIT 12.3).

\section{Materials}

Acetone

Nitrogen

Diisopropylethylamine (DIPEA)

Acetonitrile, anhydrous

4-Nitrophenyl chloroformate or acryloyl chloride

Dichloroethane, dry

Amine compound-e.g., tetraethylenepentamine;

1,4-bis-(3-aminopropoxy)butane; 4-aminomethyl-1,8,-octadiamine;

4,7,10-trioxa-1,13-tridecandiamine; $N, N$-dimethyl-1,6-hexadiamine;

2-(2-aminoethoxy)ethanol; jeffamine; 3-amino-1,2-propandiol

Dimethylformamide (DMF), dry and amine free

Methanol, reagent grade

$18 \times 8-\mathrm{cm}$ polypropylene vessels with tight-fitting lids

Solid support medium (e.g., glass, polypropylene foil, nylon membrane, polypropylene membrane) with anchoring groups in place on surface (e.g., see Support Protocol 1 for silanizing glass)

Orbital shaker

\section{Activate solid support}

1. Clean an $18 \times 8-\mathrm{cm}$ polypropylene vessel carefully with acetone and dry thoroughly.

Any polypropylene vessel can be used if it is of sufficient size to act as a reaction container for the derivatization of the support medium in bulk. It must also have a lid that fits tightly to prevent moisture from entering the reaction chamber. An $18 \times 8$-cm container can be used to process up to six microscope slides in parallel or one $\sim 17 \times 7.5-\mathrm{cm}$ sheet of polypropylene foil, and will have a wash volume of $\sim 30$ to $40 \mathrm{ml}$.

2. Flush the container with nitrogen and fill with a solution of $171 \mu \mathrm{l}(1 \mathrm{mmol})$ DIPEA in $30 \mathrm{~mL}$ anhydrous acetonitrile.

3. Add one of the following:

$192 \mathrm{mg}(1 \mathrm{mmol})$ 4-nitrophenyl chloroformate

$81 \mu \mathrm{l}(1 \mathrm{mmol})$ acryloyl chloride.

See Background Information for a discussion of these activating agents. 
4. Add the solid support medium (with anchoring groups) and close the lid carefully to seal the reaction chamber.

5. Incubate $2 \mathrm{hr}$ at ambient temperature on an orbital shaker.

6. Remove the reaction solution, wash the supports thoroughly (two times) with 30 to $40 \mathrm{ml}$ dichloroethane, and then dry under a flush of nitrogen.

It is not advisable to store the activated supports. It is best to proceed directly to the reaction with the amine component.

\section{React with amine}

7. Clean a second polypropylene container carefully with acetone and dry thoroughly.

8. Flush the container with nitrogen and fill it with a solution of $1 \mathrm{mmol}$ of the desired amine component in $30 \mathrm{~mL}$ anhydrous amine-free DMF.

In principle, any amine reagent can be employed in this reaction (see Commentary and materials section for suggestions).

9. Add the activated solid support medium to the amine reaction solution and close the lid carefully to seal the reaction chamber.

10. Incubate at ambient temperature on an orbital shaker for $12 \mathrm{hr}$ if 4-nitrophenyl chloroformate was used for activation or $24 \mathrm{hr}$ if acryloyl chloride was used.

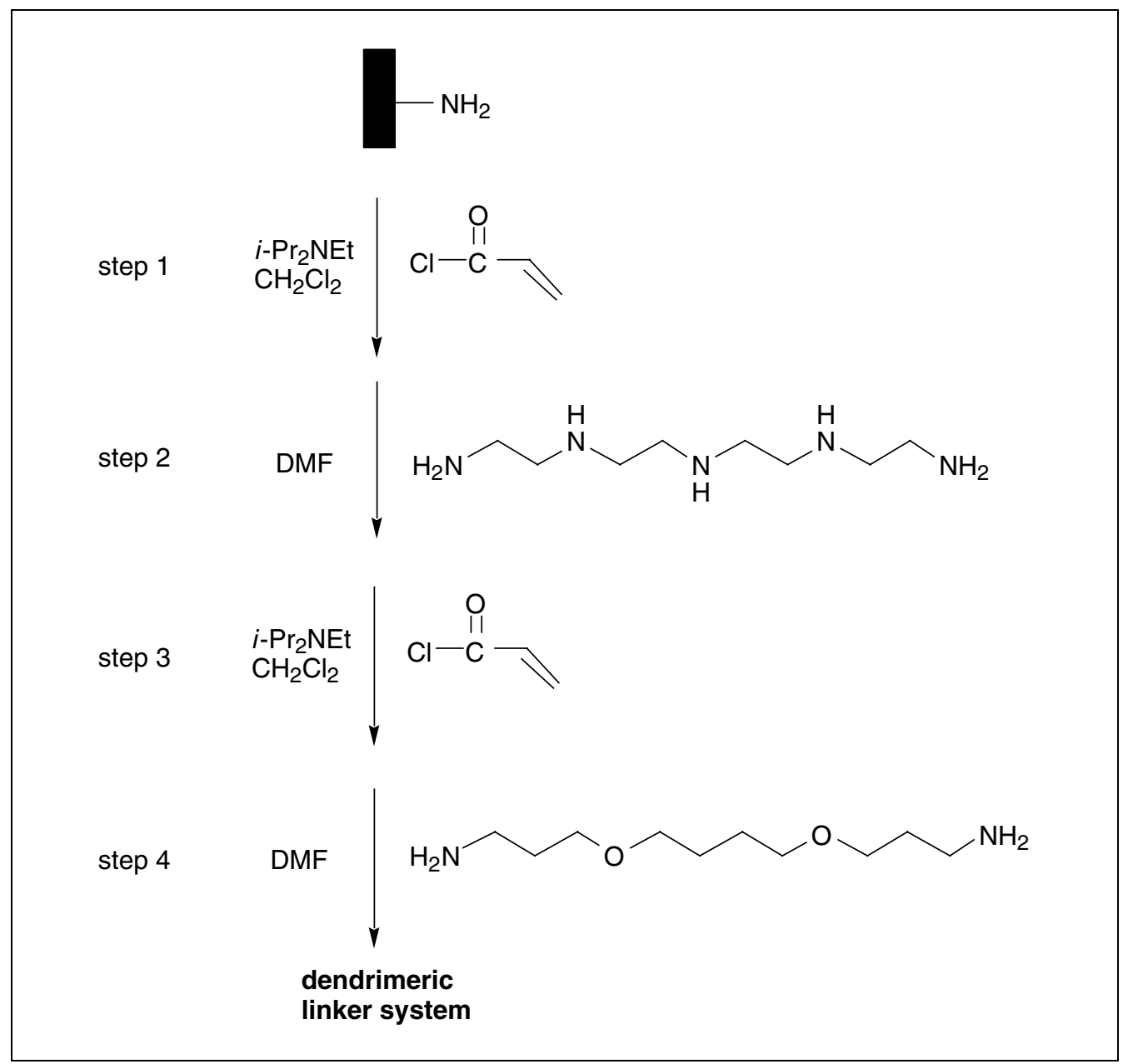

Figure 12.4.2 By an iterative process of activation and amination, linker structures of different complexity and diverse inherent characteristics can be synthesized.

Nucleic

Acid-Based

Microarrays and Nanostructures

12.4.3 
SUPPORT PROTOCOL 1

The nucleophilic substitution of the 4-nitrophenyl moiety by an amine produces a colored group, which allows monitoring of the reaction by visual inspection.

11. Remove the reaction solution and wash the support media thoroughly with DMF, then methanol, and finally acetone. Dry under a flush of nitrogen.

12. If desired, perform another round of activation and amine reaction in order to increase surface loading/dendrimeric character of the support or to alter the surface properties.

For the derivatization of microarray glass surfaces used for the immobilization of nucleic acids or in situ synthesis of DNA oligonucleotides, the best results are obtained with two reaction cycles consisting of acryloyl chloride (activation), tetraethylenepentamine (amine derivatization), acryloyl chloride (activation), and 1,4-bis-(aminopropoxy)butane (amine derivatization). This is illustrated in Figure 12.4.2.

13. Store derivatized support in a dry place up to several weeks at $4{ }^{\circ} \mathrm{C}$

\section{SILANIZATION OF GLASS SLIDES}

Prior to the actual silanization, the glass is etched, thereby improving the overall quality of the process. Both processes are described below.

\section{Materials}

$10 \%(\mathrm{w} / \mathrm{v})$ aqueous $\mathrm{NaOH}$

$1 \%(\mathrm{v} / \mathrm{v})$ aqueous $\mathrm{HCl}$

Methanol, reagent grade

$3 \%(\mathrm{w} / \mathrm{v})$ aminopropyltrimethoxysilane in $95 \%(\mathrm{v} / \mathrm{v})$ methanol

Nitrogen

Glass microscope slides (e.g., Menzel-Gläser, Germany)

Several glass vessels with lids (e.g., hematology staining jar)

Orbital shaker

Polypropylene vessel with lid

Bath sonicator

$110^{\circ} \mathrm{C}$ oven

\section{Etch glass surface}

1. Immerse underivatized glass microscope slides in a glass vessel filled with a $10 \%$ (w/v) $\mathrm{NaOH}$ solution and close the lid.

Etching can be performed in the type of glass staining jar frequently used in hematology for labeling experiments. These allow eight to ten slides to be placed standing within the vessel.

2. Shake gently overnight on an orbital shaker.

3. Remove slides from the $\mathrm{NaOH}$ bath using tweezers.

4. Wash by immersing the slides in a glass vessel filled with water and shaking briefly ( 2 to $3 \mathrm{~min}$ ) on an orbital shaker.

5. Wash in another glass vessel, filled with $1 \%(\mathrm{v} / \mathrm{v}) \mathrm{HCl}$, by shaking 2 to $3 \mathrm{~min}$ on an orbital shaker.

6. Wash 2 to $3 \mathrm{~min}$ in water.

7. Immerse the slides in a glass vessel filled with methanol and shake 2 to $3 \mathrm{~min}$ on an orbital shaker. Proceed directly to the silanization reaction.

\section{Derivatization of Glass and Polypropylene Surfaces}

\subsection{4}


8. Fill a polypropylene container with $3 \%(\mathrm{w} / \mathrm{v})$ aminopropyltrimethoxysilane in $95 \%$ $(\mathrm{v} / \mathrm{v})$ methanol.

9. Immerse etched glass slides in the container so that they just cover the bottom in a single layer. Close the lid.

A single layer is used to prevent adherence between the slides.

10. Transfer the container to a bath sonicator. Sonicate $15 \mathrm{~min}$ at ambient temperature.

CAUTION: The reaction mixture becomes hot during sonication. Open lid carefully.

11. Remove slides with tweezers.

12. Wash by immersing the slides in a glass vessel filled with methanol and shaking 2 to $3 \mathrm{~min}$ on an orbital shaker. Use very gentle agitation to prevent the slides from moving and adhering to one another.

13. Wash 2 to $3 \mathrm{~min}$ in water on an orbital shaker.

14. Dry slides under a stream of nitrogen gas.

15. Transfer slides to a preheated $110^{\circ} \mathrm{C}$ oven for $15 \mathrm{~min}$.

16. Remove slides from oven and store up to several weeks at a convenient temperature.

\section{QUALITY CONTROL OF DERIVATION REACTIONS}

To control the efficiency of the derivatization procedure, a bromphenol blue staining reaction is employed. The quality is assessed by measuring the amount of surface-bound amino functions and hence the surface loading. This assay is preferably carried out on a control strip made of the appropriate support material and added to the derivatization reactions. After each amination step, a part of the control strip is removed and analyzed.

For glass supports, only qualitative assessments can be made because of the low loading capacity of glass. Because the blue color achieved with glass can barely be visualized by eye, a polypropylene control strip is used in the same reactions with the glass slides. If a strong loading (blue color) is achieved on the polypropylene control strip, it can be assumed that the derivatization reaction in general was successful and that the slides are usable.

Quantitative assessments can be made with polypropylene foils and membranes, and with nylon membranes, all of which have sufficiently high loading to obtain reliable readings by spectrophotometry. For quantitative purposes, the control strip must be of the identical support medium used for derivatization.

\section{Additional Materials (also see Basic Protocol)}

Control strip: hydroxylated or aminated solid support (see Basic Protocol 1)

Amine-free DMF containing 0.05\% bromphenol blue

Ethanol, reagent grade

$20 \%$ piperidine in DMF

Spectrophotometer, $605 \mathrm{~nm}$

1. Add a control strip to the reactions described in the Basic Protocol. For quantification, use the identical support material that was derivatized for use.

2. At each step in the derivatization, remove an $\sim 1-\mathrm{cm}^{2}$ portion of the control strip.

For quantification and comparison purposes, always take identical portions.

SUPPORT

PROTOCOL 2

Nucleic

Acid-Based

Microarrays and

Nanostructures

\subsection{5}

Supplement 18 
3. Transfer to a new vessel and wash four times with 10 to $15 \mathrm{~mL}$ amine-free DMF.

4. Transfer to $2 \mathrm{~mL}$ amine-free DMF containing $0.05 \%(\mathrm{w} / \mathrm{v})$ bromphenol blue and incubate 5 to $10 \mathrm{~min}$ with gentle agitation.

In the presence of amino functions on the support surface, the support will turn blue and the staining solution will turn yellow.

5. Transfer to a new vessel and wash three times with ethanol to remove any residual stain.

6. Transfer to $20 \%$ piperidine in amine-free DMF to destain. Agitate gently until the blue color of the control strip is completely removed.

7. Collect the destain solution and analyze in a spectrophotometer at $605 \mathrm{~nm}\left(\varepsilon_{605}=\right.$ $95,000 \mathrm{M}^{-1} \mathrm{~cm}^{-1}$ ). If necessary, dilute solution with $20 \%$ piperidine in DMF.

8. Compare the blue color/absorption of different rounds of derivatization to monitor surface loading.

For polypropylene, the actual number of amino functions per area can be calculated. For glass, only relative measurements across reactions are possible.

SUPPORT PROTOCOL 3

Derivatization of Glass and Polypropylene Surfaces

\subsection{6}

\section{IMMOBILIZATION OF 5'-AMINO-LABELED NUCLEIC ACID COMPOUNDS}

While in situ DNA synthesis can proceed directly on the linker resulting from two subsequent reaction cycles with acryloyl chloride, tetraethylenepentamine, acryloyl chloride, and 1,4-bis-(aminopropoxy)butane (see Basic Protocol), an additional activation step is required for the covalent attachment of prefabricated $5^{\prime}$-amino-labeled nucleic acid compounds (oligonucleotides, PCR products, peptide nucleic acids).

\section{Additional Materials (also see Basic Protocol)}

Phenylene diisothiocyanate (PDITC) or dimethylsuberimidate dihydrochloride (DMS)

$10 \%(\mathrm{v} / \mathrm{v})$ anhydrous pyridine in amine-free DMF (for PDITC)

Saturated aqueous sodium bicarbonate $\left(\mathrm{NaHCO}_{3}\right.$; for DMS)

5'-Amino-labeled nucleic acid

Diisopropylethylamine (DIPEA)

$1 \mathrm{mM}$ Tris $\cdot \mathrm{Cl}, \mathrm{pH} 7.5$ (see APPENDIX 2A; optional)

6-Amino-1-hexanol

Amino-functionalized glass slides or polypropylene sheets (see Basic Protocol) $37^{\circ} \mathrm{C}$ humid chamber

\section{Activate linker}

For activation with PDITC

1a. Fill an $18 \times 8-\mathrm{cm}$ polypropylene container with $192 \mathrm{mg}(1 \mathrm{mmol})$ PDITC in $40 \mathrm{~mL}$ of $10 \%(\mathrm{v} / \mathrm{v})$ anhydrous pyridine in DMF.

2a. Immerse amino-functionalized glass slides or polypropylene sheets in activation solution and incubate for $2 \mathrm{hr}$ with gentle agitation on an orbital shaker.

3a. Wash twice with 30 to $40 \mathrm{~mL}$ amine-free DMF, shaking gently for 2 to 3 min each.

4a. Wash twice as above with dichloroethane.

5a. Dry slides under a stream of nitrogen gas. 
1b. Fill an $18 \times 8-\mathrm{cm}$ polypropylene container with $273 \mathrm{mg}(1 \mathrm{mmol}) \mathrm{DMS}$ in $40 \mathrm{~mL}$ saturated $\mathrm{NaHCO}_{3}$.

2b. Immerse the amino-functionalized glass slides or polypropylene sheets in activation solution and incubate for $1 \mathrm{hr}$ with gentle agitation on an orbital shaker.

3b. Wash twice with 30 to $40 \mathrm{~mL}$ water, shaking gently for 2 to 3 min each.

4b. Wash twice as above with acetone.

5b. Dry slides under a stream of nitrogen gas.

\section{Spot nucleic acids onto activated surfaces}

6. Take up a $5^{\prime}$-amino-labeled nucleic acid in $1 \%(\mathrm{w} / \mathrm{v})$ DIPEA in water or $1 \mathrm{mM}$ Tris.Cl, $\mathrm{pH} 7.5$.

7. Administer droplets of 0.1 to $50 \mathrm{nl}$ onto the activated support.

8. Incubate in a humid chamber at $37^{\circ} \mathrm{C}$ overnight.

9. Wash twice with 30 to $40 \mathrm{~mL}$ water, shaking gently for 2 to 3 min each.

10. Wash twice as above with methanol.

11. Deactivate by incubating $2 \mathrm{hr}$ in a freshly made solution of $50 \mathrm{mM} 6$-amino-1-hexanol and $150 \mathrm{mM}$ DIPEA in DMF.

12. Wash the DNA arrays successively (two times each) with DMF, acetone, and water.

13. Store arrays dry for 4 to 8 weeks at $4^{\circ} \mathrm{C}$.

\section{COMMENTARY}

\section{Background Information}

This unit describes the synthesis of a flexible linker system on glass and polypropylene support media (Beier and Hoheisel, 1999). The reaction scheme consists of a two-step procedure: an initial activation followed by a reaction with an amine, preferentially a polyamine. In the first step, a surface-bound anchoring group is reacted with 4-nitrophenyl chloroformate to form an activated ester, or with acryloyl chloride to form an $\alpha, \beta$-unsaturated carbonyl derivative (Fig. 12.4.1). Both products represent activated species that are reactive towards an amine derivative. The reaction of the nitrophenyl ester with an amine proceeds quickly, whereas the $\alpha, \beta$-unsaturated carbonyl derivative needs a prolonged 24-hr reaction time. However, because the latter leads to better final hybridization results, activation with acryloyl chloride is preferred.

After having completed a first cycle of derivatization (activation plus amine coupling), the very same procedure may be repeated with the same or a different amine reagent. By simple variation of the amino components, different linker types can be synthesized. The linker properties can be mod- ulated in many ways, from merely increasing the distance between the support surface and the biomolecule to be attached, to multiplying the loading capacity. If a polyamine such as tetraethylenepentamine is utilized, for example, the loading capacity of the support should be increased approximately five-fold. Since polyamines contain both primary and secondary amino functions, one unique product is not generated, but rather a mixture of compounds. Furthermore, the number of amino functions incorporated during linker synthesis controls the number of positive charges at neutral $\mathrm{pH}$. Employing the amines found in the materials section of the Basic Protocol, a large number of different linker types have been synthesized by this reaction scheme (Beier and Hoheisel, 1999). The support characteristics can be tailored to the requirements of the intended applications.

Many linker systems were found to be well suited for binding nucleic acids; however, one system in particular proved to be excellent under all conditions and was used for attaching prefabricated DNA oligonucleotides, PCR products, and peptide nucleic acid (PNA) oligomers, as well as for in situ synthesis of
Nucleic

Acid-Based

Microarrays and Nanostructures

12.4.7 
DNA microarrays. It is generated preferably on aminated (glass) surfaces by sequential reactions with acryloyl chloride, tetraethylenepentamine, acryloyl chloride, and 1,4-bis(aminopropoxy)butane (Fig. 12.4.2). For in situ synthesis of DNA microarrays (Pease et al., 1997; Beier and Hoheisel, 2000), no further derivatization step is required. The terminal amino group of the linker serves directly as the starting point for oligonucleotide synthesis.

For immobilization of prefabricated nucleic acids, an additional activation is needed to generate a covalent linkage between biomolecule and support. For that purpose, bifunctional cross-linking reagents like phenylene diisothiocyanate or dimethylsuberimidate are utilized (Beier and Hoheisel, 1999). Activating the solid-phase surface prevents any crossreaction between the nucleic acids prior to immobilization. Prior to spotting onto the support, a base is added to the nucleic acid solution, since the cross-linking agents have their optimal reactivity in a basic milieu. Because of its non-nucleophilic character, diisopropylethylamine is employed, since it does not compete for the reactive sites on the support. However, immobilization also works at neutral $\mathrm{pH}$ (i.e., in water). Since small droplets evaporate quickly after application, the reaction is run to completion by incubating the DNA arrays in a humid chamber at $37^{\circ} \mathrm{C}$ after administration of the spots. Alternatively, betaine may be used to reduce evaporation (Diehl et al., 2001).

\section{Critical Parameters}

Overall, the procedure is very robust. The most critical parameter in actual routine processing is preventing moisture from entering the reaction chamber during the two-step derivatization. Also, there should be no lengthy time interval between activation and amination.

\section{Anticipated Results}

Building a dendrimeric structure increases the loading capacity significantly, which can be a critical factor for glass supports in particular. With the protocols for coupling nucleic acids as detailed above, an increase by ten-fold has been observed.

\section{Time Considerations}

The overall preparation time depends on the number of iterative activation and amination steps performed and is mainly determined by the incubations times. About 1 day should be considered for each cycle of activation and amination. However, even if several iterative cycles are performed, throughput is no major limitation, since surface modification can be performed in bulk.

\section{Literature Cited}

Beier, M. and Hoheisel, J.D. 1999. Versatile derivatisation of solid support media for covalent bonding on DNA-microchips. Nucl. Acids Res. 27:1970-1977.

Beier, M. and Hoheisel, J.D. 2000. Production of quantitative photolithographic synthesis of individually quality checked DNA microarrays. Nucl. Acids Res. 28:e11.

Diehl, F., Grahlmann, S., Beier, M., and Hoheisel, J.D. 2001. Manufacturing DNA-microarrays of high spot homogeneity and reduced background signal. Nucl. Acids Res. 29:e38.

Pease, A.C., Solas, D., Sullivan, E.J., Cronin, M.T., Holmes, C.P., and Fodor, S.P.A. 1994. Lightgenerated oligonucleotide arrays for rapid DNA sequence analysis. Proc. Natl. Acad. Sci. U.S.A. 91:5022-5026.

\section{Contributed by Markus Beier}

Febit AG

Mannheim, Germany

Jörg D. Hoheisel

Deutsches Krebsforschungszentrum

Heidelberg, Germany

\section{Derivatization of Glass and Polypropylene Surfaces}

12.4.8 